\title{
Development, evaluation and validation of a screening tool for late onset bacteremia in neonates - a pilot study
}

Sandra A. N. Walker ${ }^{1,2^{*}}$ (D), Melanie Cormier ${ }^{1}$, Marion Elligsen ${ }^{1}$, Julie Choudhury ${ }^{3}$, Asaph Rolnitsky ${ }^{3}$, Carla Findlater ${ }^{3}$ and Dolores laboni ${ }^{3}$

\begin{abstract}
Background: Clinical and laboratory parameters can aid in the early identification of neonates at risk for bacteremia before clinical deterioration occurs. However, current prediction models have poor diagnostic capabilities. The objective of this study was to develop, evaluate and validate a screening tool for late onset ( $>72 \mathrm{~h}$ post admission) neonatal bacteremia using common laboratory and clinical parameters; and determine its predictive value in the identification of bacteremia.

Methods: A retrospective chart review of neonates admitted to a neonatal intensive care unit (NICU) between March 1, 2012 and January 14, 2015 and a prospective evaluation of all neonates admitted between January 15, 2015 and March 30, 2015 were completed. Neonates with late-onset bacteremia (> $72 \mathrm{~h}$ after NICU admission) were eligible for inclusion in the bacteremic cohort. Bacteremic patients were matched to non-infected controls on several demographic parameters. A Pearson's Correlation matrix was completed to identify independent variables significantly associated with infection ( $p<0.05$, univariate analysis). Significant parameters were analyzed using iterative binary logistic regression to identify the simplest significant model $(p<0.05)$. The predictive value of the model was assessed and the optimal probability cut-off for bacteremia was determined using a Receiver Operating Characteristic curve.
\end{abstract}

Results: Maximum blood glucose, heart rate, neutrophils and bands were identified as the best predictors of bacteremia in a significant binary logistic regression model. The model's sensitivity, specificity and accuracy were 90 , 80 and $85 \%$, respectively, with a false positive rate of $20 \%$ and a false negative rate of $9.7 \%$. At the study bacteremia prevalence rate of $51 \%$, the positive predictive value, negative predictive value and negative post-test probability were 82,89 and $11 \%$, respectively.

Conclusion: The model developed in the current study is superior to currently published neonatal bacteremia screening tools. Validation of the tool in a historic data set of neonates from our institution will be completed.

Keywords: Neonates, Late onset bacteremia, Screening tool

\footnotetext{
* Correspondence: sandra.walker@sunnybrook.ca

'Department of Pharmacy E-302, Sunnybrook Health Sciences Centre (SHSC),

2075 Bayview Avenue, Toronto, ON M4N 3M5, Canada

${ }^{2}$ Leslie Dan Faculty of Pharmacy, University of Toronto, Toronto, Ontario,

Canada

Full list of author information is available at the end of the article
}

(c) The Author(s). 2019 Open Access This article is distributed under the terms of the Creative Commons Attribution 4.0 International License (http://creativecommons.org/licenses/by/4.0/), which permits unrestricted use, distribution, and reproduction in any medium, provided you give appropriate credit to the original author(s) and the source, provide a link to the Creative Commons license, and indicate if changes were made. The Creative Commons Public Domain Dedication waiver (http://creativecommons.org/publicdomain/zero/1.0/) applies to the data made available in this article, unless otherwise stated. 


\section{Background}

The increased risk of late-onset infections (greater than $72 \mathrm{~h}$ following birth) in preterm and very low birth weight (VLBW) neonates is well documented [1]. Despite advancements in care, late-onset sepsis occurs in up to $20 \%$ of VLBW infants, with $28 \%$ of septic neonates experiencing more than one episode [2].

The diagnosis of late-onset neonatal sepsis is reached using various signs and symptoms, and often leads to the initiation of empiric, broad spectrum antimicrobial therapy before laboratory results are available [2]. In a study by Wirschafter et al., it was found that the ratio of antibiotic courses administered to the number of confirmed blood stream infections (BSIs) in neonates was $14: 1$, suggesting that antibiotic overuse is an issue that needs to be addressed in this patient population [3].

The reason for antibiotic overuse in the neonatal population is multifactorial. The lack of specificity of symptoms of bacteremia and the overlap of shared symptoms among various neonatal conditions produces an extensive list of differential diagnoses for clinicians to consider and may lead to the overuse of broad-spectrum antibiotics. Because the sensitivity of laboratory diagnosis of BSIs in neonates is affected by the small volumes of blood permissible in blood draws $(0.5 \mathrm{~mL})$, clinicians cannot rely on blood cultures alone, with false negative rates of up to $60 \%$ in low colony count sepsis [4]. Currently, healthcare professionals in the neonatal intensive care unit (NICU) lack a standardized, validated prediction tool for bacteremia. Published screening tools that predict bacteremia have deficiencies in their performance metrics (e.g. sensitivity and specificity) which limit their application in clinical practice [5-14].

In addition to common clinical [15] and laboratory parameters that are used to subjectively predict bacteremia and sepsis in neonates, acute phase reactants such as Creactive protein (CRP) and procalcitonin (PCT) [16, 17] are being investigated; however, they have limitations [17] and are either not routinely measured or quickly available in most hospitals. Similarly, although the intercellular messenger CD64 has been shown to be an accurate diagnostic marker of early- and late-onset neonatal sepsis [18], it is not routinely measured in clinical practice. Other novel predictors of infection have also surfaced [16, 19-23], and although the investigation of these new biomarkers as predictors of neonatal sepsis is exciting and may be promising in the future, they are unavailable to clinicians today.

Given the rate of antibiotic use in the NICU, a practical screening tool for bacteremia would enable safer, more appropriate use of antibiotics. An ideal screening tool for bacteremia in neonates should provide sufficient sensitivity to ensure a case of bacteremia is not missed, with a low negative post-test probability so as to promote a decrease in empiric, broad spectrum antimicrobials in non-bacteremic neonates. The objective of this study was to develop, evaluate and validate a screening tool for late onset ( $>72 \mathrm{~h}$ post admission) neonatal bacteremia using common laboratory and clinical parameters.

\section{Methods \\ Study design}

This pilot study was approved with the need for informed consent waived by the Sunnybrook Health Sciences Centre (SHSC) Research Ethics Board on January 13, 2015. The study employed a prospective and retrospective study design. The retrospective cohort of neonates included all eligible patients admitted to the institution's 48 bed level 3 NICU from March 1, 2012 to January 14, 2015. The prospective cohort of neonates were all eligible patients admitted to the institution's NICU from January 15, 2015 to April 30, 2015.

All neonates admitted to the institution's NICU during the study period were eligible for study inclusion, regardless of gestational age. Neonates that did not have at least some relevant laboratory parameters or vital signs collected during their stay were excluded, as they did not have data to contribute to the development of the screening tool. This included neonates admitted to the NICU for hyperbilirubinemia and hypoglycemia (unrelated to sepsis) who only had laboratory monitoring of bilirubin and/or blood glucose, as well as neonates staying in the NICU for less than $48 \mathrm{~h}$ who did not have laboratory parameters or vital signs collected, recorded, or accessible to the team collecting data. Only neonates with late-onset bacteremia (bacteremia occurring greater than $72 \mathrm{~h}$ after admission to the NICU) were eligible for study inclusion in the case cohort.

\section{Data collection}

Data on 35 clinical parameters and 17 laboratory parameters were collected for bacteremic cases and controls (retrospective and prospective cohorts) (Additional file 1: Table S1). These parameters were selected based on previously established and hypothesized potential signs and symptoms of bacteremia in neonates. Data for the retrospective component of the study were obtained from archived charts in the SHSC Health Records Office, the Electronic Patient Record (EPR), and the antimicrobial stewardship database.

For the prospective component, the clinical and laboratory parameter data were collected daily by a team of NICU pharmacists for all patients included in the study from date of NICU admission (day 0) to the date of first positive blood culture, discharge from the NICU, or death (whichever came first). 
Following data collection, neonates with documented bacteremia (cases) were matched to non-infected neonates (controls) to reduce the risk of differences in baseline characteristics with univariate analysis having some unknown confounding effect on parameters that may influence the diagnosis of bacteremia. The identification of significant parameters with univariate analysis and subsequent confirmation with Pearson's correlation provided justification of parameter entry into the binary logistic regression. At the point of data entry into the binary logistic regression there is no further importance related to matching; and therefore, relevant data from both matched and unmatched controls were eligible for tool development using binary logistic regression to maximize sample size. Control patients were neonates who did not receive antibiotics during their NICU hospital admission beyond the first $48 \mathrm{~h}$ of life and never had a positive culture at any site. Cases were matched to controls based on gender (when possible), gestational age at birth, corrected gestational age at study entry, weight at study entry, total length of stay in the NICU, and antibiotic use (yes or no) within the first $48 \mathrm{~h}$ of life. The remainder of control patients were categorized as unmatched controls.

Neonates were categorized as having late-onset bacteremia if a blood culture was positive for noncontaminant bacteria more than $72 \mathrm{~h}$ into their NICU admission. Neonates with blood isolates considered to represent contaminants (Corynebacterium spp., Propionibacterium spp., and Bacillus species other than B. anthracis [24]) were excluded from further comparative analysis of bacteremic versus non-bacteremic patients to avoid any potential confounding. The criteria for a true coagulase negative Staphylococcus spp. (CONS) infection in neonates varies $[25,26]$, therefore for the purpose of the current study, neonates found to have blood cultures positive for CONS were included as bacteremic cases for analysis if the colony count was reported as greater than 100 colonies or if appropriate antibiotics were used for 7 or more days in response to the positive culture and correlated clinical status of the patient. If the colony count for CONS was less than 10 colonies or antibiotics were used for less than 7 days in response to the positive culture, the neonate was excluded from analysis.

At the time of their first positive non-contaminant blood culture, neonates were classified as cases and matched one-to-one to controls for analysis. The time of the positive blood culture represented the time of study entry for bacteremic cases. In the event that a patient had multiple positive blood cultures during their NICU hospital stay, data was only collected in relation to their first positive blood culture identified $>72 \mathrm{~h}$ into their NICU admission.
The data collected for final analyses were the parameter results closest to but before the date of blood culture collection within the previous $24 \mathrm{~h}$ period in cases and the variable result closest to the matching length of stay day post-birth within the previous $24 \mathrm{~h}$ period for controls (i.e. if case patient had positive blood culture $96 \mathrm{~h}$ after birth, then relevant parameters for case and their matched control patient were obtained from $72 \mathrm{~h}$ to $96 \mathrm{~h}$ after birth). In the case of laboratory parameters that were infrequently ordered (CRP and lactate), the respective closest value within a period of $96 \mathrm{~h}$ before the blood culture collection date (in cases) or matched days post-birth (in controls) was recorded. In the case of clinical parameters in which a maximum or minimum value was needed, the parameters were defined as being the maximum or minimum within $24 \mathrm{~h}$ before the date of blood culture collection in cases or matched days post birth in controls. Data on unmatched controls were obtained from the neonate's worst day in the NICU using fraction of inspired oxygen ( $\mathrm{FiO} 2)$ as the marker given the highest priority for determining worst NICU day. For neonates who were not ventilated and on room air, the worst NICU day was the day with the most out of range clinical or laboratory parameters.

\section{Data analysis \\ Sample size}

In the literature, there is currently no standard ratio to determine how many patients are required per independent variable analyzed in the development of a screening tool. Traditionally, minimum ratios from 2:1 to $10: 1$ (patients to variables), and a minimum sample size of 100-200 patients has been considered acceptable [27-32]. A target sample size of 100 neonates would allow for assessment of a maximum of 10 (at a ratio of 10:1) up to 50 (at a ratio of 2:1) variables for association with bacteremia in the evaluation to create a screening tool. A total of 52 clinical and laboratory parameters were included for potential assessment in the current study. If each of these parameters was significant with univariate analysis and entered into the iterative binary logistic regression modelling, a minimal sample size of 104 neonates (for a ratio of 2 patients to 1 variable) would be required.

\section{Statistics}

Descriptive statistics (mean with standard deviation or median, and range or percentage) were used to describe patient characteristics. Univariate analyses using a twotailed unpaired t-test (interval data normally distributed), two-tailed unpaired t-test with Welch correction for normally distributed data with unequal standard deviations; Mann-Whitney U test (interval data not normally distributed, or ordinal data), or Fisher's Exact Test and 
odds ratios with 95\% confidence interval (nominal data) (GraphPad Instat, version 3.05, 32 bit for WIN 95/NT, created September 27, 2000) were used to compare patient characteristics, clinical parameters, and laboratory values obtained from cases versus controls. One-way analysis of variance (ANOVA) (interval parametric data) and Kruskal-Wallis (interval nonparametric data) were used when comparing characteristics across $>2$ groups of patients. A Pearson's Correlation matrix (SPSS version 13.0 for Windows, created September 1, 2004) was completed to identify clinical and laboratory parameters (independent variables) associated with bacteremia (dependent variable) (thereby, confirming the univariate analyses) and to determine the percentage of patients with a given measured variable. Any clinical and laboratory parameters available for $>20 \%$ of patients and having a $p$ value $<0.05$ with both univariate analysis and Pearson's Correlation were entered into binary logistic regression (SPSS version 13.0 for Windows, created September 1,2004$)$ using an iterative process to identify a statistically significant model $(p<0.05)$ in which all independent variables remaining in the model had an odds ratio of $>1$ and which provided the highest sensitivity and specificity. Only patients with a complete data set for the identified significant independent variables were included in the development of the final model. A Receiver Operating Characteristic (ROC) curve was developed to identify the optimal probability breakpoint representing bacteremia. Classification and Regression Tree Analysis (CART) (Salford Predictive Modeler 7.0 Pro $32 \mathrm{mb}$ ) was used to identify breakpoints of each independent variable that remained significant in the final model. Sensitivity and specificity analysis was conducted on the best predictive model for bacteremia. The optimal bacteremia screening tool developed was compared to published tools by mapping the sensitivity and false positive rate (1-specificity) for all tools to generate a ROC curve.

\section{Results}

A total of 2214 neonates were admitted to the NICU between March 1, 2012 - March 31, 2015 and 153 of these neonates (7\%) (42 cases, 42 matched controls, 69 prospective unmatched controls, 111 total controls) were included in this study (Fig. 1). Patient characteristics of the entire study population $(n=153)$ and patient characteristics of the sample of patients that had a complete data set for inclusion in the development of the final bacteremia screening tool (cases $=31$, controls $=30$ ) are detailed in Additional file 1: Tables S2 and S3, respectively. The overall period prevalence of bacteremia at the study hospital during the study period was $2 \%(42 / 2214)$. Six of 111 control patients (5\%) (including 3 matched control patients $(3 / 42,7 \%))$ had blood cultures drawn and processed, each of which was negative for any microbial growth. One of these control patients had complete data and was included in tool development (1/30, 3\%). The majority of organisms isolated in blood samples for bacteremic cases were Gram Positive bacteria (38 out of 45 isolates, 84\%) (Additional file 1: Table S4).

The 26 parameters found to be significantly correlated with bacteremia by univariate analysis are detailed in Additional file 1: Table S5. Significant parameters that were identified in univariate analysis, but were not input into the iterative binary logistic regression process were: mortality that was possibly related to bacteremia, survival at the end of NICU stay, number of days in NICU and number of ventilation days, since these would not be parameters known to a clinician at the time of using the screening tool in clinical practice, and therefore would not be helpful in a predictive tool; maximum mean arterial pressure (MAP) was excluded because there is no normal range in babies and it is influenced by corrected gestational age; all parameters with a significant negative correlation (gestational age at birth, corrected gestational age at entry, weight at entry, minimum temperature, and maximum serum creatinine) were excluded because they would not be helpful in a predictive tool to identify bacteremia. Therefore, of the original 26 significant parameters identified by univariate analysis, only 16 parameters were assessed in the iterative binary logistic regression. Sixty-one neonates had a complete data set for inclusion in the development of the optimal binary logistic regression model (31 cases, 30 controls). Therefore, the patient to variable ratio for the iterative binary logistic regression process was 4:1, which is considered acceptable [27-32]. Of the cases included in the final data set for tool development, 29 were from the retrospective chart review and 2 were from the prospective chart review. Of the controls included in the final data set for tool development, 2 were matched controls from the retrospective chart review and 28 were unmatched controls from the prospective chart review. The remaining neonates with missing clinical and/or laboratory values were excluded $(n=92 ; 10$ cases, 82 controls).

The optimal binary logistic regression model for the bacteremia screening tool (Table 1) was Ln (odds of bacteremia) $=-25.459+0.752$ (Maximum Blood Glucose $[\mathrm{mmol} / \mathrm{L}])+0.119$ (Maximum Heart Rate $[\mathrm{bpm}])+0.108(\%$ Bands) +0.071 (Maximum Neutrophils $[\times 10\langle 9\rangle / \mathrm{L}])$.

Therefore, odds of bacteremia is the exponential of the preceding equation and the probability of bacteremia $=$ Odds of Bacteremia/ (1+ Odds of Bacteremia). Using a ROC curve, the optimal probability cut-off for bacteremia (i.e. the threshold above which a neonate would be deemed to be bacteremic) was found to be $>$ $41.5 \%$ with an area under the curve of $89 \%$. The CART 


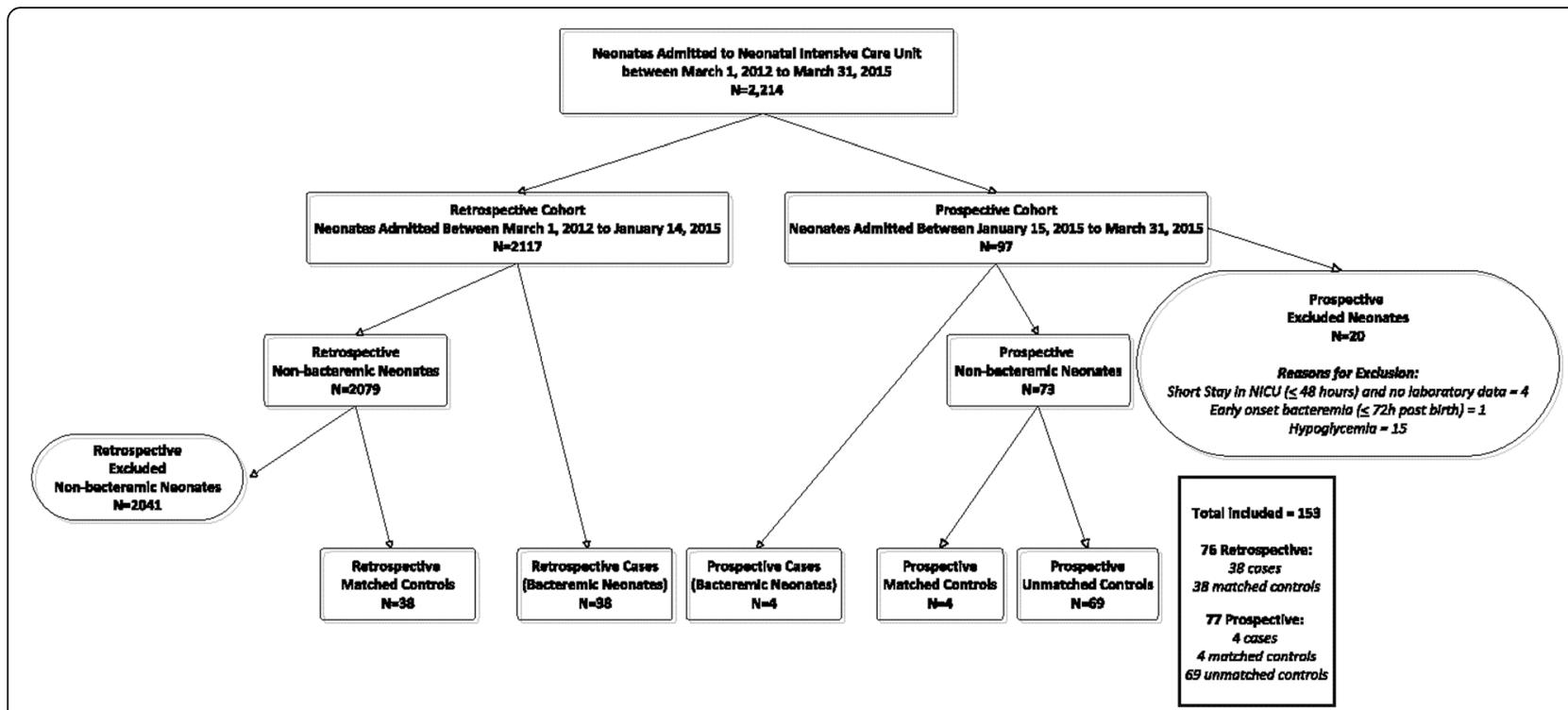

Fig. 1 Patient Eligibility Flow Chart

determined breakpoints for the parameters in the bacteremia screening tool are detailed in Table 1.

The optimal model has a sensitivity of $90 \%$ (false negative rate of $10 \%$ ), a specificity of $80 \%$ (false positive rate of $20 \%$ ), and an overall accuracy of $85 \%$. Positive and negative likelihood ratios were 4.50 and 0.12 respectively. The screening tool's positive predictive value (PPV) was $82 \%$, and the negative predictive value (NPV) was $89 \%$. At the study population's pre-test probability of $51 \%$, the screening tool had a negative post-test probability of $11 \%$. At the overall study period prevalence of bacteremia of $2 \%$, this translates to a negative post-test

Table 1 Optimal model for Bacteremia in neonates

\begin{tabular}{|c|c|c|c|}
\hline \multicolumn{4}{|c|}{ Binary logistic regression analysis } \\
\hline \multicolumn{4}{|c|}{$\begin{array}{l}\text { Ln (Odds Bacteremia }(Y / N)=-25.459+0.752 \text { (Maximum Blood Glucose } \\
[\mathrm{mmol} / \mathrm{L}])+0.119(\text { Maximum Heart Rate }[\mathrm{bpm}])+ \\
0.108(\% \text { Bands })+0.071\left(\text { Maximum Neutrophils }\left[\times 10^{9} / \mathrm{L}\right]\right)\end{array}$} \\
\hline \multicolumn{4}{|c|}{ Variables in Final Binary Logistic Regression Equation } \\
\hline $\begin{array}{l}\text { Independent } \\
\text { Variable }\end{array}$ & $\begin{array}{l}\text { Odds } \\
\text { Ratio }\end{array}$ & $\begin{array}{l}95 \% \\
\text { Confidence } \\
\text { Interval }\end{array}$ & $\begin{array}{l}\text { CART breakpoint for } \\
\text { association with bacteremia } \\
\text { when parent node is maximum } \\
\text { blood glucose }\end{array}$ \\
\hline $\begin{array}{l}\text { Maximum } \\
\text { Blood Glucose } \\
(\mathrm{mmol} / \mathrm{L})\end{array}$ & 2.121 & $1.182-3.806$ & $>6$ \\
\hline $\begin{array}{l}\text { Maximum } \\
\text { Heart Rate } \\
\text { (bpm) }\end{array}$ & 1.127 & $1.040-1.221$ & $>186$ \\
\hline$\%$ Bands & 1.114 & $0.574-2.160$ & $>2.15$ \\
\hline $\begin{array}{l}\text { Maximum } \\
\text { Neutrophils } \\
\left(\times 10^{9} / \mathrm{L}\right)\end{array}$ & 1.073 & $0.932-1.236$ & $>11.7$ \\
\hline
\end{tabular}

probability of $0.2 \%$ (Additional file 1: Table S6). Importantly, Additional file 1: Table S6 could be used by clinicians and investigators: i) to identify the predicted PPV, $\mathrm{NPV}$, and negative post-test probability of our tool at the bacteremia prevalence (pre-test probability) in their hospital and ii) to compare our tool to other published tools reporting a different bacteremia prevalence. When compared to other screening tools using a ROC curve, our model had the lowest false-positive rate while maintaining a high sensitivity (Fig. 2 and Table 2).

The screening tool developed in this pilot study was validated in a small separate retrospective cohort of neonates admitted to the NICU between September 12, 2010 and February 29th, 2012 with a full data set for the tool parameters (unpublished data) $(n=8$; bacteremic neonates, $n=7$; non-bacteremic neonates, $n=1$ ) (Additional file 1: Table S7). The tool identified all 7 bacteremic neonates and differentiated the nonbacteremic neonate from the group.

\section{Discussion}

A screening tool that accurately predicts the probability of late-onset bacteremia in neonates using four parameters (blood glucose, heart rate, bands, and neutrophils) that are readily available through routine blood work and monitoring in the NICU was developed. In the developmental cohort, the tool has a sensitivity of $90 \%$ (false negative rate of $10 \%$ ), a specificity of $80 \%$ (false positive rate of $20 \%$ ), an accuracy of $85 \%$, a positive and negative likelihood ratio were 4.50 and 0.12 respectively, a positive predictive value of $82 \%$, a negative predictive value of $89 \%$, and at the study population's pre-test probability of $51 \%$, the screening tool had a negative 


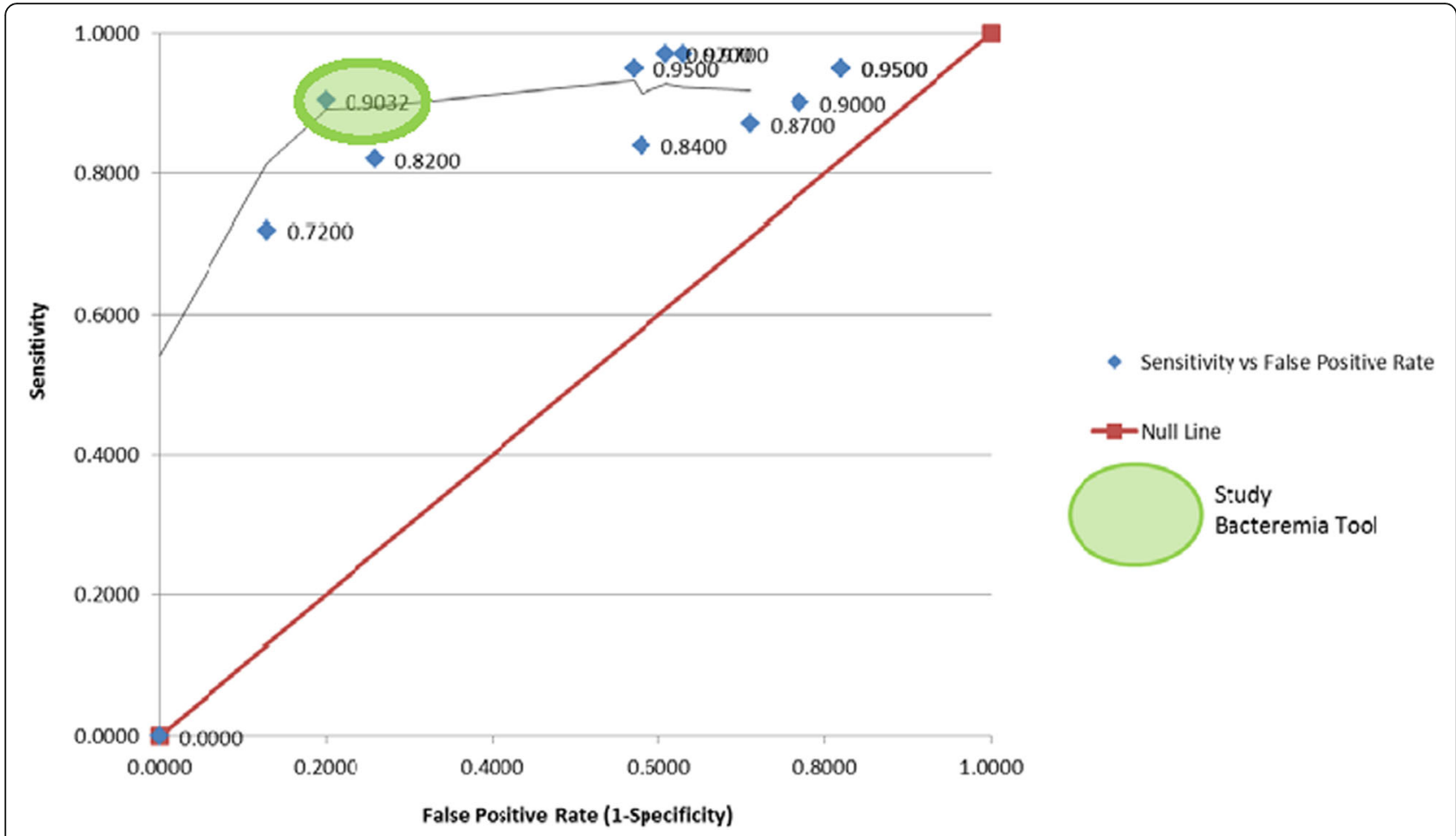

Fig. 2 Receiver Operating Characteristic Curve Comparing Study Bacteremia Screening Tool to Currently Published Screening Tools [5-14]

post-test probability of $11 \%$. At the overall hospital study period prevalence of bacteremia during the study period of $2 \%$, this translates to a negative post-test probability of $0.2 \%$, meaning that the risk of missing a neonate with true bacteremia is $<1 \%$ at the study bacteremia prevalence. A user-friendly app can be accessed at https://sun nybrook.ca/content/?page=antimicrobial-stewardship-

blood-screening-neo and is available at no charge for clinical use to provide clinicians with a fast calculation of the probability of BSI (\%) in their patients and make recommendations for obtaining blood cultures and consideration of empiric antimicrobial management based on practical probability cut-offs (Additional file 2: Figure S1).

The tool developed in this study had the lowest false positive rate while maintaining a high sensitivity (Fig. 2) compared to previously published tools [5-14]. In addition, when an equal period prevalence was used to compare the tools, our study tool had a negative posttest probability that was equal to or lower than previously published screening tools with better overall metrics for sensitivity and specificity [5-14] (Table 2). Mahieu et al. in 2000, developed a screening tool with high sensitivity and low negative post-test probability that assigns points if various clinical and laboratory parameters, including CRP, polymorphonuclear neutrophil (PMN) fraction, temperature, number of days of Total Parenteral Nutrition (TPN), and platelet count, exceed a certain threshold [7]. The model's performance was tested at various cut-off points, with a score of 8 or greater having the highest sensitivity and lowest negative post-test probability. Despite the screening tool's excellent sensitivity, its ability to differentiate between bacteremic and non-bacteremic neonates is poor, with a specificity of only 43\% [7] .

Despite the high sensitivity of some previously developed screening tools $[5-8,11,13]$, their low specificity would result in an inability to differentiate between bacteremic and non-bacteremic neonates. While the priority is to detect all neonates with bacteremia, a tool that over-selects for bacteremia is of little use clinically.

Our study was not without limitations. Given that a portion of our study was retrospective, there is a potential for confounding factors to impact outcomes; however, we hope that the incorporation of a prospective component has minimized any confounding. Furthermore, we were unable to collect complete data sets for all neonates due to the observatory nature of the study design. The inability to collect complete data sets may have impacted on our ability to evaluate parameters which were not often obtained (e.g. change in level of consciousness, liver function tests, arterial lactate, venous lactate, and albumin). Since we only included neonates with full data sets in the final analysis to create our model, our final sample size was reduced from 153 to 61 , which may have influenced our ability to identify significant parameters with Pearson's correlation (univariate 
Table 2 Performance comparison of study developed tool to currently published screening tools [5-14]

\begin{tabular}{|c|c|c|c|c|c|c|c|c|c|}
\hline Sensitivity & Specificity & $\begin{array}{l}\text { Period } \\
\text { Prevalence }\end{array}$ & $\begin{array}{l}\text { Positive } \\
\text { Post-Test } \\
\text { Probability }\end{array}$ & $\begin{array}{l}\text { Negative } \\
\text { Post-Test } \\
\text { Probability }\end{array}$ & $\begin{array}{l}\text { Positive } \\
\text { Likelihood } \\
\text { Ratio }\end{array}$ & $\begin{array}{l}\text { Negative } \\
\text { Likelihood } \\
\text { Ratio }\end{array}$ & $\begin{array}{l}\text { False } \\
\text { Positive } \\
\text { rate }\end{array}$ & $\begin{array}{l}\text { False } \\
\text { Negative } \\
\text { Rate }\end{array}$ & $\begin{array}{l}\text { Study Developed } \\
\text { Tool Negative Post } \\
\text { Test Probability at } \\
\text { Citation Bacteremia } \\
\text { Period Prevalence }\end{array}$ \\
\hline
\end{tabular}

\begin{tabular}{|c|c|c|c|c|c|c|c|c|c|c|}
\hline \multicolumn{11}{|c|}{ Study Developed Tool } \\
\hline$P$ value $>0.415$ & 0.90 & 0.80 & 0.51 & 0.82 & 0.11 & 4.50 & 0.12 & 0.20 & 0.10 & - \\
\hline \multicolumn{11}{|l|}{$\begin{array}{l}\text { Mahieu et al., } \\
2000 \text { [7] }\end{array}$} \\
\hline Score $\geq 8$ & 0.95 & 0.43 & 0.41 & 0.54 & 0.08 & 1.67 & 0.12 & 0.57 & 0.05 & 0. \\
\hline Score $\geq 11$ & 0.60 & 0.84 & 0.41 & 0.72 & 0.25 & 3.75 & 0.48 & 0.16 & 0.40 & \\
\hline Score $\geq 14$ & 0.26 & 1.00 & 0.41 & 1.00 & 0.34 & 9999.00 & 0.74 & 0.00 & 0.74 & 0 \\
\hline Score $\geq 11$ & 0.72 & 0.87 & 0.41 & 0.79 & 0.18 & 5.50 & 0.32 & 0.13 & 0.28 & \\
\hline
\end{tabular}

plus

positive

culture

Mahieu et al.,

2002 [5]

\begin{tabular}{|c|c|c|c|c|c|c|c|c|c|}
\hline Score $\geq 11$ & 0.84 & 0.42 & 0.55 & 0.64 & 0.32 & 1.45 & 0.38 & 0.58 & 0.16 \\
\hline Score $\geq 11+3$ RFs & 0.82 & 0.67 & 0.55 & 0.75 & 0.25 & 2.48 & 0.27 & 0.33 & 0.18 \\
\hline
\end{tabular}

Singh et al.,

2003 [8]

\begin{tabular}{|c|c|c|c|c|c|c|c|c|c|}
\hline Score $\geq 1$ & 0.87 & 0.29 & 0.29 & 0.33 & 0.16 & 1.23 & 0.45 & 0.71 & 0.13 \\
\hline Score $\geq 2$ & 0.53 & 0.80 & 0.29 & 0.52 & 0.19 & 2.65 & 0.59 & 0.20 & 0.47 \\
\hline
\end{tabular}

Okascharoen et al., 2005 [9]

$$
\begin{aligned}
& \text { Score } \geq 4 \\
& \text { Score } \geq 5 \\
& \text { Score } \geq 6 \\
& \text { Dalgic et al., } \\
& 2006 \text { [10] }
\end{aligned}
$$

$0.82 \quad 0.74$

\subsection{7}

0.39

0.05

3.15

0.24

$\begin{array}{ll}0.26 & 0.18\end{array}$

0.02

$0.70 \quad 0.82$

0.17

0.44

0.07

3.89

0.37

$0.18 \quad 0.30$

0.02

$0.47 \quad 0.96$

0.17

0.71

0.10

12.00

0.55

$0.04 \quad 0.53$

0.02

Score $=6-12$

0.56

0.71

0.39

0.55

0.28

1.93

0.62

0.29

0.44

0.07

Okascharoen et al., 2007 [6]

Validation
Cohort
Score $\leq 3$
(low risk of
sepsis)
Validation
Cohort
Score 4-7
(medium
risk of
sepsis)
Validation
Cohort
Score $\geq 8$
(high risk of
sepsis)

Score $\leq 3$

(low risk of

Validation

Score 4-7

(medium

risk of

sepsis)

Validation

\begin{tabular}{|c|c|c|c|c|c|c|c|c|c|c|}
\hline $\begin{array}{l}\geq 1 \text { clinical } \\
\text { signs }\end{array}$ & 0.90 & 0.23 & 0.27 & 0.30 & 0.14 & 1.17 & 0.43 & 0.77 & 0.10 & 0.04 \\
\hline$\geq 2$ clinical & 0.52 & 0.65 & 0.27 & 0.36 & 0.21 & 1.49 & 0.74 & 0.35 & 0.48 & 0.04 \\
\hline
\end{tabular}

Score $\geq 8$

$$
\text { sepsis) }
$$

Kudawla et al., 2008 [11] 
Table 2 Performance comparison of study developed tool to currently published screening tools [5-14] (Continued)

\begin{tabular}{|c|c|c|c|c|c|c|c|c|c|c|}
\hline & Sensitivity & Specificity & $\begin{array}{l}\text { Period } \\
\text { Prevalence }\end{array}$ & $\begin{array}{l}\text { Positive } \\
\text { Post-Test } \\
\text { Probability }\end{array}$ & $\begin{array}{l}\text { Negative } \\
\text { Post-Test } \\
\text { Probability }\end{array}$ & $\begin{array}{l}\text { Positive } \\
\text { Likelihood } \\
\text { Ratio }\end{array}$ & $\begin{array}{l}\text { Negative } \\
\text { Likelihood } \\
\text { Ratio }\end{array}$ & $\begin{array}{l}\text { False } \\
\text { Positive } \\
\text { rate }\end{array}$ & $\begin{array}{l}\text { False } \\
\text { Negative } \\
\text { Rate }\end{array}$ & $\begin{array}{l}\text { Study Developed } \\
\text { Tool Negative Post- } \\
\text { Test Probability at } \\
\text { Citation Bacteremia } \\
\text { Period Prevalence }\end{array}$ \\
\hline \multicolumn{11}{|l|}{ signs } \\
\hline$\geq 2$ markers & 0.48 & 0.70 & 0.27 & 0.37 & 0.21 & 1.60 & 0.74 & 0.30 & 0.52 & 0.04 \\
\hline $\begin{array}{l}\geq 1 \text { clinical sign }+ \\
\geq 2 \text { markers }\end{array}$ & 0.95 & 0.18 & 0.27 & 0.30 & 0.09 & 1.16 & 0.28 & 0.82 & 0.05 & 0.04 \\
\hline \multicolumn{11}{|l|}{$\begin{array}{l}\text { Rosenberg et al., } \\
2010 \text { [12] }\end{array}$} \\
\hline Score $\geq 1$ & 0.77 & 0.50 & 0.54 & 0.64 & 0.35 & 1.54 & 0.46 & 0.50 & 0.23 & 0.12 \\
\hline Score $\geq 2$ & 0.42 & 0.82 & 0.54 & 0.73 & 0.45 & 2.33 & 0.71 & 0.18 & 0.58 & 0.12 \\
\hline \multicolumn{11}{|l|}{ Bekhof et al., 2013 [13] } \\
\hline 1 of 4 signs present & 0.97 & 0.37 & 0.27 & 0.36 & 0.03 & 1.54 & 0.08 & 0.63 & 0.03 & 0.04 \\
\hline
\end{tabular}

analysis) and the model development with iterative binary logistic regression. Lastly, neonates with a positive blood culture growing CONS, bacteria typically considered to be contaminants when isolated in the adult population, were included or excluded from the study based on a combination of culture result and clinical judgement. The partially subjective nature of this approach to inclusion or exclusion of a neonate from the study, although not ideal, is difficult to avoid even in a purely prospective study in neonates, due to the subjective current approach to treatment of CONS bacteremia in neonates $[25,26]$.

\section{Conclusions}

A clinical tool that can be used at the bedside to determine the probability that a neonate has late-onset bacteremia could assist clinicians in the decisionmaking process when it comes to requesting blood cultures and initiating broad-spectrum antibiotics in the NICU. The screening tool developed in this study incorporates four parameters that are readily available to clinicians through routine monitoring and standard care. Whereas current screening tools aim only to detect bacteremia, our tool has the potential capacity to differentiate between bacteremic and non-bacteremic neonates - a feature that could be of significant value to clinicians who are deciding whether to draw blood cultures or initiate broad spectrum antibiotics in the event of negative blood cultures. While the results of the preliminary validation of our tool in a small retrospective sample of neonates were encouraging, prospective validation of the screening tool in a larger sample size is required and is planned at the study site.

\section{Additional files}

Additional file 1 : Table S1. Clinical and Laboratory Data Collection Parameters. Table S2. Patient Characteristics of Entire Study Population ( $N=153)$. Table S3. Characteristics of Patients Included in Final Bacteremia Tool. Table S4. Microbiological Characteristics in Blood Cultures. Table S5. Parameters Significantly Associated with Bacteremia (Univariate Analysis). Table S6. What Would Happen with Bacteremia Tool if Pre-Test Probability were Different?. Table S7. Patient

Characteristics of Validation Cohort $(N=8)$. (DOCX $47 \mathrm{~kb})$

Additional file 2 : Figure S1. Screening tool for early identification of bloodstream infection in neonates. This figure provides a screenshot of the screening tool. (TIF $887 \mathrm{~kb}$ )

\section{Abbreviations}

ANOVA: Analysis of variance; BSI(s): Blood stream infection(s); CART: Classification and Regression Tree; CONS: Coagulase negative Staphylococcus spp; CRP: C-reactive protein; EPR: Electronic Patient Record; FiO2: Friction of inspired oxygen; MAP: Mean arterial pressure; NICU: Neonatal intensive care unit; NPV: Negative predictive value; PCT: Procalcitonin; PMN: Polymorphonuclear neutrophil; PPV: Positive predictive value; ROC: Receiver Operating Characteristic; SHSC: Sunnybrook Health Sciences Centre; TPN: Total Parenteral Nutrition; VLBW: Very low birth weight

\section{Acknowledgments}

None.

\section{Authors' contributions}

SANW: conceived the project idea, was the senior investigator contributing to and overseeing all phases of this research (protocol, conduct, data analysis, manuscript). MC: was involved in all phases of the study (protocol, conduct, data analysis, manuscript). ME: contributed to the protocol and manuscript. JC: assisted with data collection, protocol and manuscript. AR: contributed to the protocol and manuscript. CF: assisted with data collection, protocol and manuscript. DI: assisted with data collection, protocol and manuscript. All Authors read and approved the manuscript.

Funding

No funding was obtained for any component of this study. 


\section{Availability of data and materials}

The datasets used and/or analysed during the current study are available from the corresponding author on reasonable request.

\section{Ethics approval and consent to participate}

This study was approved with the need for informed consent waived by the Sunnybrook Health Sciences Centre (SHSC) Research Ethics Board on January 13, 2015.

\section{Consent for publication}

Not applicable.

\section{Competing interests}

The authors declare that they have no competing interests.

\section{Author details}

'Department of Pharmacy E-302, Sunnybrook Health Sciences Centre (SHSC), 2075 Bayview Avenue, Toronto, ON M4N 3M5, Canada. ${ }^{2}$ Leslie Dan Faculty of Pharmacy, University of Toronto, Toronto, Ontario, Canada. ${ }^{3}$ SHSC, Women and Babies Program, Toronto, Ontario, Canada.

Received: 13 April 2019 Accepted: 16 July 2019

Published online: 24 July 2019

\section{References}

1. Plano LRW. The changing spectrum of neonatal infectious disease. J Perinatol. 2010;30:S16-20.

2. Stoll BJ, Hansen N, Fanaroff AA, Wright LL, Carlo WA, Ehrenkranz RA, et al. Late-onset Sepsis in very low birth weight neonates: the experience of the NICHD neonatal research network. Pediatr. 2002;110(2):285-91.

3. Wirtschafter DD, Padilla G, Wan K, Trupp D, Fayard EES. Antibiotic use for presumed neonatally acquired infections far exceeds that for central lineassociated blood stream infections: an exploratory critique. J Perinatol. 2011; 31:514-8.

4. Schelonka RL, Chai MK, Yoder BA, Hensley D, Brockett RM, Ascher DP. Volume of blood required to detect common neonatal pathogens. J Pediatr. 1996;129(2):275-8.

5. Mahieu LM, De Dooy JJ, Cossey VR, Goosens LL, Vrancken SL, Jespers AY, et al. Internal and external validation of the NOSEP prediction score for nosocomial sepsis in neonates. Crit Care Med. 2002:30(7):1459-66.

6. Okascharoen C, Hui C, Cairnie J, Morris AM, Kirpalani H. External validation of bed side prediction score for diagnosis of late-onset neonatal sepsis. J Perinatol. 2007:27:496-501.

7. Mahieu LM, De Muynck AO, De Dooy JJ, Laroche SM, Van Acker KJ. Prediction of nosocomial sepsis in neonates by means of a computerweighted bedside scoring system (NOSEP score). Crit Care Med. 2000;28(6): 2026-33.

8. Singh SA, Dutta S, Narang A. Predictive clinical scores for diagnosis of late onset neonatal septicemia. J Trop Pediatr. 2003:49(4):235-9.

9. Okascharoen C, Sirinavin S, Thakkinstian A, Kitayaporn D, Supapanachart S. A bedside prediction-scoring model for late onset neonatal Sepsis. J Perinatol. 2005;25(12):778-83.

10. Dalgic N, Ergenekon E, Koc E, Atalay Y. NOSEP and clinical scores for nosocomial sepsis in a neonatal intensive care unit (letter). J Trop Pediatr. 2006;52(3):226-7.

11. Kudawla M, Dutta S, Narang A. Validation of a clinical score for the diagnosis of late onset neonatal septicemia in babies weighing 1000-2500 g. J Trop Pediatr. 2008:54(1):66-9.

12. Rosenberg RE, Ahmed ASMNU, Saha SK, Chowdhury MAKA, Ahmed S, Law $\mathrm{PA}$, et al. Nosocomial sepsis risk score for preterm infants in low resource settings. J Trop Pediatr. 2010;56(2):82-9.

13. Bekhof J, Reitsma JB, Kok JH, Van Straaten IHLM. Clinical signs to identify late-onset sepsis in preterm infants. Eur J Pediatr. 2013;172(4):501-8.

14. Verstraete EH, Blot $K$, Mahieu L, Vogelaers D, Blot S. Prediction models for neonatal health care-associated sepsis: a meta-analysis. Pediatrics. 2015; 135(4):e1002-14

15. Yapicioglu H, Ozlu F, Sertdemir Y. Are vital signs indicative for bacteremia in newborns? J Matern Fetal Neonatal Med. 2015;28(18):2244-9.

16. Oeser C, Lutsar I, Metsvaht T, Turner MA, Heath PT, Sharland M. Clinical trials in neonatal sepsis. J Antimicrob Chemother. 2013;68:2733-45.
17. Ng PC. Diagnostic markers of infection in neonates. Arch Dis Child Fetal Neonatal Ed. 2003:89:F229-F35.

18. Streimish I, Bizzarro M, Northrup V, Wang C, Renna S, Koval N, et al. Neutrophil CD64 with hematologic criteria for diagnosis of neonatal Sepsis. Am J Perinatol. 2014;31:21-30.

19. Adly AAM, Ismail EA, Andrawes NG, El-Saadany MA. Circulating soluble triggering receptor expressed on myeloid cells-1 (STREM-1) as diagnostic and prognostic marker in neonatal sepsis. Cytokine. 2014;65:184-91.

20. Sarafidis K, Soubasi-Griva V, Piretzi K, Thomaidou A, Agakidou E, Taparkou A, et al. Diagnostic utility of elevated serum soluble triggering receptor expressed on myeloid cells (STREM)-1 in infected neonates. Intensive Care Med. 2010;36:864-8

21. Prashant A, Vishwanath P, Kulkarni P, Sathya NP, Gowdara V, Nataraj SM, et al. Comparative assessment of cytokines and other inflammatory markers for the early diagnosis of neonatal sepsis-a case control study. PLoS One. 2013;8(7):e68426. https://doi.org/10.1371/journal.pone.0068426.

22. Gokmen Z, Ozkiraz S, Kulaksizoglu S, Kilicdag H, Ozel D, Ecevit A, Tarcan A. Resistin-a novel feature in the diagnosis of sepsis in premature neonates. Am J Perinatol. 2013;30(6):513-8.

23. Suguna Narasimhulu S, Hendricks-Munoz KD, Borkowsky W, Mally P. Usefulness of urinary immune biomarkers in the evaluation of neonatal sepsis: a pilot project. Clin Pediatr. 2013:52(6):520-6.

24. Weinstein MP. Blood culture contamination: persisting problems and partial Progress. J Clin Microbiol. 2003:41(6):2275-8.

25. Venkatesh MP, Placencia F, Weisman LE. Coagulase-negative staphylococcal infections in the neonate and child: an update. Semin Pediatr Infect Dis. 2006:17:120-7

26. Isaacs D. A ten year, multicentre study of coagulase negative staphylococcal infections in Australasian neonatal units. Arch Dis Child Fetal Neonatal Ed. 2003:88:F89-93.

27. Anderson T, Rubin H. Statistical inference in factor analysis. Berkeley: University of California Press; 1956.

28. Everitt BS. Multivariate analysis: the need for data, and other problems. Br J Psychiatry. 1975;126:237-40.

29. Gorsuch R. Factor analysis. Second ed. Hillsdale: Lawrence Erlbaum Associates; 1983

30. Kline P. A handbook of test construction: introduction to psychometric design. London: Methuen and Co; 1986

31. Nunnally J. Psychometric theory. Second ed. New York: McGraw-Hill; 1978.

32. Velicer W, Fava J. Effects of variable and subject sampling on factor pattern recovery. Psychol Methods. 1998;3:231-51.

\section{Publisher's Note}

Springer Nature remains neutral with regard to jurisdictional claims in published maps and institutional affiliations.

Ready to submit your research? Choose BMC and benefit from:

- fast, convenient online submission

- thorough peer review by experienced researchers in your field

- rapid publication on acceptance

- support for research data, including large and complex data types

- gold Open Access which fosters wider collaboration and increased citations

- maximum visibility for your research: over $100 \mathrm{M}$ website views per year

At BMC, research is always in progress.

Learn more biomedcentral.com/submission 